\title{
Experiência do Estudante de Semiologia Médica em Aulas Práticas com o Paciente à Beira do Leito
}

\section{PALAVRAS-CHAVE \\ - Educação Médica \\ - Currículo \\ - Ensino.}

\section{KEYWORDS:}

- Education, Medical

- Curriculum

- Teaching.

\author{
Medical Students' Experience in Practical \\ Classes on Bedside Clinical Examination
}

Rilva Lopes de Sousa-Muñoz Isabel Barroso Augusto Silva José Luis Simões Maroja

\section{RESUMO}

O objetivo deste estudo foi identificar as percepções de estudantes de Medicina que cursaram a disciplina de Semiologia Médica em relação ao contato com os pacientes à beira do leito em aulas práticas no Hospital Universitário Lauro Wanderley, da Universidade Federal da Paraíba. Pesquisa observacional e descritiva com entrevistas por meio de autoaplicação de questionário semiestruturado elaborado pelos autores e pré-testado. Participaram do estudo 105 alunos com idade entre 20 e $24(21 \pm 1,1)$ anos, $56,2 \%$ do sexo masculino. A grande maioria declarou dificuldade na abordagem clínica inicial do doente $(89 / 84,8 \%)$ e $72(68,6 \%)$ referiram insegurança ou medo. Houve aumento de respostas positivas em momentos posteriores da disciplina, porém $34,3 \%$ ainda reportaram sentimentos negativos. Observou-se maior constrangimento no questionamento do paciente sobre temas mais intimos por alunos do sexo masculino. Esses achados reforçam a necessidade de valorizar questões éticas, humanísticas e psicopedagógicas no início da graduação nas escolas médicas.

\section{ABSTRACT}

This study focused on the perceptions of medical students in the course on Clinical Examination in relation to contact with patients during bedside practical classes at the Lauro Wanderley University Hospital, Federal University in Paraíba, Brazil. The observational and descriptive study included a self-applied semi-structured questionnaire prepared and pretested by the authors. The study sample included 105 medical students ranging from 20 to 24 years of age (21 \pm 1.1$), 56.2 \%$ of whom were males. The vast majority $(89$, or $84.8 \%$ ) reported difficulty in their initial clinical approach to patients, and $72(68.6 \%)$ reported insecurity or fear. There was an increase in positive responses at later moments during the course, but $34.3 \%$ still reported negative feelings. Male students were more embarrassed when asking patients about intimate matters. These findings highlight the need to value ethical, humanistic, and psychopedagogical issues during undergraduate medical training.
Recebido em: 24/05/2010

Reencaminhado em: 24/01/2011 Reencaminhado em: 21/02/2011 Aprovado em: 11/03/2011 


\section{INTRODUÇÃO}

A disciplina de Semiologia Médica é a introdução à fase clínica da graduação e, portanto, o contato inicial do aluno de Medicina com o paciente. Trata-se de um momento importante de construção de uma teoria e de uma prática capazes de preparar o aluno para o cuidado ao paciente, abordando aspectos essenciais da arte médica.

O objetivo principal da disciplina é treinar os alunos de Medicina nas técnicas básicas de entrevista e exame físico dos pacientes. A Semiologia proporciona também o aprendizado dos primórdios da relação médico-paciente e se articula com todas as demais disciplinas médicas, representando um marco na entrada para o ciclo profissional do curso.

O programa prático da disciplina na Universidade Federal da Paraíba (UFPB) requer aulas de iniciação ao exame clínico à beira do leito e treinamento do aluno no exame físico dos pacientes internados nas enfermarias de Clínica Médica do Hospital Universitário Lauro Wanderley (HULW). Diferentemente da pedagogia geral, que repousa em um processo ensino-aprendizagem polarizado por apenas dois focos, o professor e o aluno, na pedagogia médica devem ser levados em conta três focos - professor, aluno e paciente-, com naturezas, expectativas e papéis diferentes ${ }^{1}$.

Com base nessas considerações, o objetivo principal deste estudo foi identificar a percepção discente sobre a interação estudante-paciente na fase de aprendizado prático inicial do exame clínico nas enfermarias do HULW/UFPB.

\section{METODOLOGIA}

Trata-se de uma pesquisa observacional e descritiva, de natureza exploratória, envolvendo alunos que cursaram a disciplina de Semiologia Médica (Departamento de Medicina Interna, Centro de Ciências Médicas da UFPB) nos períodos letivos de 2008.1 e 2008.2, de currículo tradicional.

Os alunos foram entrevistados no início do semestre seguinte ao do término da disciplina. Os critérios de inclusão foram: ter concluído a disciplina de Semiologia Médica e assinar Termo de Consentimento para participar da pesquisa. O projeto foi aprovado pelo Comitê de Ética em Pesquisa do HULW/UFPB.

A técnica de coleta de dados foi a entrevista direta, com autoaplicação de um questionário semiestruturado elaborado pelos autores e pré-testado ${ }^{2}$. Os estudantes foram contatados nas salas de aula por alunos monitores de Semiologia Médica. A aplicação dos questionários durou aproximadamente 15 minutos. Os alunos responderam aos questionários individualmente sem discussão entre eles, entregando-os aos monitores imediatamente após o término do preenchimento.
O questionário destinado à entrevista com o aluno consistiu em dados demográficos e perguntas fechadas sobre sua experiência pessoal ao cursar a disciplina. As perguntas versaram sobre dificuldades sentidas na abordagem clínica inicial do paciente, sentimentos apresentados perante o exame clínico, receptividade do paciente, opinião sobre a participação de pacientes nas aulas, repercussão das aulas práticas sobre estes, reações próprias diante de desconfortos apresentados pelo paciente, entre outras questões relacionadas.

Empregou-se principalmente a estatística descritiva na análise dos dados. Na estatística inferencial, usaram-se técnicas não paramétricas de análise (qui-quadrado para dados categóricos e teste de Mann-Whitney para dados quantitativos).

\section{RESULTADOS}

Participaram do estudo 105 alunos, com idade entre 20 e 24 anos $(21,0 \pm 1,1), 59(56,2 \%)$ do sexo masculino.

A grande maioria relatou dificuldade na abordagem clínica inicial do doente $(89 / 84,8 \%)$, e $72(68,6 \%)$ referiram insegurança ou medo.

Ao concluírem a disciplina, 76 (72,4\%) afirmaram sentimentos positivos ao terem que realizar o exame clínico (satisfação, confiança, curiosidade), enquanto 36 (34,3\%) ainda reportaram sentimentos negativos (insegurança, medo) (Tabela 1). Não houve associação estatística entre relato de dificuldade na abordagem inicial do paciente e gênero, com proporção semelhante de homens e mulheres na distribuição desta variável.

TABELA 1

Sentimentos declarados pelos alunos $(n=105)$ ao final da disciplina de Semiologia Médica do DMI/CCM/UFPB nos períodos 2008.1 e 2008.2 em relação à ideia de realizar novos exames clínicos

\begin{tabular}{lcc}
\hline \multicolumn{1}{c}{ Sentimentos declarados } & $f$ & $\mathbf{\%}$ \\
\hline Satisfação & 34 & 32,4 \\
Confiança & 29 & 27,6 \\
Insegurança/medo & 25 & 23,8 \\
Curiosidade & 13 & 12,4 \\
Indisposição / aborrecimento & 6 & 5,7 \\
Indiferença & 3 & 2,8 \\
Outro (desconforto) & 2 & 1,9 \\
\hline
\end{tabular}

A maior parte dos pacientes foi considerada receptiva $(85,7 \%)$ pelos alunos. Sessenta e sete $(63,8 \%)$ alunos ponderaram que o doente não é obrigado a cooperar com o ensino pelo fato de estar internado em um hospital-escola. A maioria dos alunos acredita que, ao participarem das aulas práticas de 
Medicina, os pacientes obtêm benefícios (51/48,6\%) ou não obtêm prejuízos nem benefícios (30/28,6\%); 18 (17,1\%) pensam que o paciente tem benefícios e prejuízos; e 6 (5,7\%) consideram que os pacientes são prejudicados.

A maioria dos alunos referiu solicitar permissão do paciente antes de iniciar o exame clínico (89/84,8\%); 35 (33,3\%) relataram respostas negativas do paciente a tal solicitação. A maioria $(84 / 80 \%)$ também afirmou que preferia que o professor/monitor estivesse presente nos primeiros contatos com os pacientes nas enfermarias.

Quarenta e dois alunos (40\%) responderam que presenciaram reações de choro nos pacientes entrevistados; 64 (60,9\%) afirmaram ter sentido compaixão diante dessa ocorrência. Quarenta e três alunos (40,9\%) revelaram ter presenciado desconforto físico com piora dos sintomas nos pacientes durante as aulas práticas, momentos em que $27 / 43$ (62,7\%) referiram sentir compaixão ou tristeza; $12 / 43$ (27,9\%) sentiram medo ou culpa; e 10/43 (23,2\%) declararam "nada" ter experimentado diante da constatação da piora do doente.

Cinquenta e oito alunos $(55,2 \%)$ relataram constrangimento ao questionarem o paciente sobre sexualidade, uso de drogas ilícitas ou renda familiar. Houve associação entre esta resposta e gênero: alunos do sexo masculino relataram mais constrangimento em abordar tais temas na entrevista com o paciente do que as alunas $(p=0,01)$.

A maioria (69/65,7\%) respondeu que, se fosse necessária sua própria internação no HULW, aceitaria ser examinado por estudantes da área de saúde.

\section{DISCUSSÃO E CONCLUSÕES}

Neste trabalho, o objetivo principal foi verificar o ponto de vista do estudante de Medicina que cursou a disciplina de Semiologia Médica acerca de seus primeiros contatos com o paciente. Em que pesem as avaliações sobre a necessidade de contato precoce do aluno com o paciente e com a atividade profissional precípua do médico, os estudantes de Medicina do currículo tradicional não têm contato com pacientes durante os dois primeiros anos de curso na maioria das universidades brasileiras, nas quais ainda vigora, em geral, a estrutura de ciclos básico e clínico, o que deve favorecer a ocorrência de sentimentos negativos em relação ao primeiro contato com o paciente, como observado no presente estudo.

A chegada do aluno à fase clínica do curso, com o contato com o paciente hospitalizado, dá-se no quarto período do curso de Medicina, provocando geralmente sentimentos de ansiedade e medo ${ }^{3,4}$. Os contatos, até então com animais de laboratório, cadáveres e tubos de ensaio, serão agora com pessoas doentes e hospitalizadas ${ }^{1}$.
Nesse sentido, os resultados do presente estudo revelam que a maioria dos alunos entrevistados relatou sentimentos de insegurança e medo nos primeiros contatos com o paciente hospitalizado. Essa falta de preparo sentida pelo estudante torna esse momento tão esperado - o primeiro contato com o paciente - uma experiência avaliada como negativa e frustrante pelo discente ${ }^{5}$.

A obtenção da história clínica, o exame físico, a referência a antecedentes e hábitos, muitas vezes de natureza íntima, são fatores que também podem criar as inquietações observadas na amostra estudada nesta pesquisa. Esse aspecto é agravado pela falta de privacidade das enfermarias. Além disso, o estudante, como adolescente que na maioria das vezes ainda é, está aprendendo a lidar com a própria sexualidade. O próprio exame físico, por meio da exposição do corpo do paciente, coloca em xeque as noções de pudor e recato do estudante ${ }^{5}$.

É cada vez mais frequente a presença de alunos muito jovens, às vezes ainda adolescentes, no curso médico, aptos intelectualmente, mas ainda sem experiência específica do ponto de vista psicossocial. A cada geração, é menor a média de idade por ocasião do ingresso do curso ${ }^{3}$. Na maioria deles, os aspectos mencionados são potencializados pelo período do ciclo de vida em que os estudantes estão contextualizados.

A necessidade do aluno de lidar sozinho com a angústia gerada na sua formação causa um prejuízo em sua aprendizagem Tem-se tratado muito sobre como o paciente é submetido a um processo de despersonalização ao entrar num hospital. Mas essa mesma preocupação com os aspectos humanos não parece ser tão intensa quando o sujeito é o estudante de Medicina ${ }^{5,6}$.

Pelo contato com o paciente, o aluno obtém dados da história do adoecer e da vida dele, o que, muitas vezes, desperta no estudante sentimentos de culpa, como se verificou nestes resultados, pois este percebe geralmente estar aprendendo sem proporcionar benefícios ao paciente, sentindo-se "invasivo $^{\prime \prime 7,8}$.

Embora sabendo que atitude nem sempre implica comportamento correspondente, cabe destacar um dado que chamou a atenção nos resultados encontrados: uma proporção não desprezível de alunos referiu indiferença diante do desconforto do paciente observado durante aulas práticas. Estas respostas insólitas podem estar relacionadas ao problema já estudado de que, ao longo do percurso nas escolas médicas, em particular no primeiro ano de contato com a realidade clínica, ocorre uma diminuição nas habilidades éticas dos estudantes ${ }^{9,10}$, especialmente uma diminuição da sensibilidade ética e da empatia ${ }^{11,12}$.

Saiyd et al. (2005) chamam este fenômeno, verificado particularmente em médicos, de "calosidade emocional", en- 
quanto Meleiro (1999) o denominou "armadura profissional"4. Tais comportamentos ajudariam os médicos a manter uma imagem de "distanciamento emocional"13.

Lind $^{14}$ estudou o desenvolvimento da competência para julgamento moral entre estudantes de vários cursos, incluindo o de Medicina, entre estudantes alemães. Concluiu que os estudantes de Medicina apresentaram regressão na competência para o julgamento moral, ao contrário de outros estudantes universitários, que apresentaram aumento notável nessa competência. Atribui esse achado ao fato de as escolas médicas ensinarem habilidades técnicas e científicas, e não valorizarem os aspectos éticos e humanos, sendo isto um problema mundial e antigo. O ensino da ética médica é considerado deficitário em muitas escolas médicas, e o despreparo nesta área causa um dilema ético nos estudantes de Medicina ${ }^{3}$.

Ribeiro e Amaral ${ }^{15}$ observaram, como professores da faculdade de Medicina da Universidade Federal de Minas Gerais, que a maioria dos estudantes tinha a atenção mais voltada ao "caso interessante" do que ao paciente, havendo certo desinteresse por aqueles que não apresentavam doença orgânica bem definida.

Nos currículos tradicionais de Medicina, há uma busca preponderante da racionalidade técnica, o que se acentua progressivamente no decorrer do curso. Esses currículos centrados no hospital, como no caso do presente estudo, podem também ser um fator importante para a manutenção do modelo biomédico baseado na racionalidade técnica. Esse modelo, por sua vez, é responsabilizado pela desumanização da medicina de modo geral.

Tal modelo centrado em aspectos apenas biomédicos tende a inibir o crescimento moral do estudante de Medicina, comprometendo sua capacidade de sentir empatia e compaixão, e a boa prática clínica não existe separada de comportamentos éticos e profissionais, que dependem de maturidade moral. Nesse sentido, o ensino do componente afetivo da comunicação com o paciente também não tem recebido a devida atenção.

Os dados relatados justificam o interesse na avaliação da atitude do estudante de Medicina, que vem se consolidando a partir do final da última década, com a socialização desse estudante entendida como incorporação de atitudes, comportamentos e valores profissionais, sendo objeto de preocupação e estudos de muitos educadores e motivando mudanças curriculares em algumas escolas médicas atualmente ${ }^{16}$.

Sabe-se que a empatia é um componente essencial de uma relação médico-paciente satisfatória, de tal modo que cultivá-la representa um dos objetivos de ensino propostos pela Association of American Medical Colleges (AAMC) para todas as escolas de Medicina americanas ${ }^{8}$. Por outro lado, também foi possível observar que muitos estudantes revelaram uma atitude mais humana diante dos pacientes com que se depararam ao longo do semestre. A maioria se identificou com o paciente ao afirmar que concordaria em ser objeto de aulas práticas caso houvesse necessidade de se internar no HULW.

Os resultados observados sugerem também que na fase inicial do treinamento clínico o aluno já começa a apreender princípios da relação médico-paciente e de ética clínica, mas deve receber apoio nessa experiência de aprendizagem. Nesse sentido, torna-se relevante investigar as necessidades do aluno para subsidiar as intervenções a ele destinadas. As angústias do estudante poderiam ser minimizadas com a criação de espaços de discussão nos quais as emoções decorrentes da formação possam ser compartilhadas ${ }^{5}$.

O ensino e a discussão de aspectos éticos deveriam ser considerados fundamentais ao se iniciar o ciclo profissionalizante. É importante inserir preceitos éticos precocemente no curso, uma tarefa que deveria envolver todos os docentes e não somente os professores de ética médica.

Mendonça et al. ${ }^{12}$ mencionam a teoria de Paul Weil de que existem duas formas de ética, que se complementam: a moralista e a espontânea. A primeira é constituída pelas informações exógenas que são internalizadas, enquanto a segunda surge no próprio indivíduo, como algo natural. Os aspectos éticos positivos demonstrados pelos estudantes entrevistados parecem ser a ética espontânea de Weil, ligada à formação e ao contexto social onde estão inseridos e não às informações obtidas no processo de aprendizagem do curso médico, uma vez que no currículo tradicional não há uma disciplina formal de ética antes do quarto período.

Também parece haver uma ênfase deficitária e inadequada nos aspectos psicológicos dos estudantes. É importante refletir sobre o despreparo que o corpo docente, os preceptores e os complexos hospitalares têm no respaldo dos problemas enfrentados pelo estudante ${ }^{3}$.

Os indícios observados de passividade frente às atividades da enfermaria, requerendo a presença do professor na abordagem inicial do paciente, podem se relacionar aos mencionados sentimentos negativos, dificultando o desenvolvimento de iniciativa do aluno. $\mathrm{O}$ estudante tende a não se colocar como um sujeito atuante na construção da relação com o paciente, comportando-se de forma passiva durante o encontro, isentando-se da responsabilidade de construir uma relação que dependa de sua iniciativa, de uma atitude ativa de abordagem $^{3,17}$.

As disciplinas clínicas do curso médico têm no seu cotidiano algumas situações peculiares que tendem a potencia- 
lizar problemas e conflitos que normalmente fazem parte da rotina de instituições que prestam atendimento de saúde à população por meio de estudantes ${ }^{18}$. Isto acontece principalmente porque as pessoas ali atendidas são colocadas, pelo menos em parte, na condição temporária de "objeto de ensino" para os futuros profissionais. É de se esperar que pacientes que sofrem manipulação excessiva em exames repetidos se tornem irritados e se mostrem refratários à abordagem estudantil.

Por outro lado, a maioria dos pacientes demonstrou receptividade em relação à participação nas aulas práticas. Estudantes de Medicina são não apenas bem aceitos, como também esperados por muitos pacientes e acompanhantes durante visitas a enfermarias de clínica médica ${ }^{19,20}$. Fora do Brasil, a percepção do paciente sobre sua participação em aulas à beira do leito também foi estudada por Lehmann et al. ${ }^{21}$, que verificaram uma resposta favorável.

Este estudo, de caráter exploratório, abre algumas perspectivas de interesse para a educação médica. Há necessidade de reflexão sobre aspectos pedagógicos de natureza ético-humanística para aprimorar a metodologia da disciplina de Semiologia Médica, não só um momento ideal para treinar a capacidade dos alunos no exame clínico, mas também uma oportunidade única de ensinar e pôr em prática a ética.

É oportuno considerar também a visão discente do ensino numa fase marcada por mudanças, como no início do contato com pacientes à beira do leito. $\mathrm{O}$ aconselhamento dos estudantes deveria fazer parte de uma orientação psicopedagógica, de forma sistemática, pelos próprios docentes de Semiologia Médica. Seria importante também oferecer ao aluno ingressante na escola de Medicina um entendimento claro e uma expectativa realista do caminho a percorrer durante a formação médica.

Podem ser feitas algumas inferências de maior interesse para o educador a partir do conjunto de respostas dos estudantes. Foi possível verificar respostas negativas ou ambivalentes sobre a abordagem inicial do paciente, sob a forma de insegurança ou medo, referidas ao início da disciplina, embora a maior parte dos pacientes tenha sido considerada receptiva aos estudantes. Houve aumento de respostas positivas em momentos posteriores do desenvolvimento da disciplina, porém $34,3 \%$ ainda reportaram sentimentos negativos. Observou-se maior constrangimento em alunos do sexo masculino ao questionarem o paciente sobre questões mais íntimas.

Espera-se que os resultados obtidos abram outras possibilidades de pesquisa. Uma pesquisa qualitativa poderia contribuir para elucidar os resultados encontrados, principalmente em relação à ambivalência em respostas relacionadas à percepção do estudante quanto à participação do paciente nas aulas práticas.

\section{REFERÊNCIAS}

1. Jardim CBV, Costa NGS, Oliveira PC, Silva VN, Rabelo YS. O papel do aluno de graduação em Medicina no atendimento a pacientes de enfermarias de longa permanência de um hospital-escola. Rev Bras Educ Med. 2008;32(1):75-82.

2. Menendez DFS, Azevedo MH, Paiva AFA, Sousa, RL, Silva IBA, Maroja JLS. Percepção do paciente na interação com o aluno de Semiologia Médica: Proposta para questionário estruturado de observação. Anais do X Encontro de Iniciação à Docência da UFPB, João Pessoa, 2007. [acesso em: 21 fev 2011]. Disponível em: http://www.prac. ufpb.br/anais/IXEnex/iniciacao/documentos/anais/6. SAUDE/6CCSDMIMT09.pdf.

3. Castro CV. Os Temores na Formação e Prática da Medicina: Aspectos Psicológicos. Rev Bras Educ Med. 2004;28(1):3845.

4. Sayd JD, Silva DA, Pinheiro MPD. O Aprendizado de Semiologia em um Currículo Tradicional. Rev Bras Educ Med. 2003;7(2):104-13.

5. Quintana, AM, Rodrigues AT, Arpini DM, Bassi LA, Cecim PS, Santos MS. A angústia na formação do estudante de medicina. Rev Bras Educ Med. 2008;32(1):7-14.

6. Taquette SR, Rego S, Schramm FR, Soares LP, Carvalho SV. Situações eticamente conflituosas vivenciadas por estudantes de Medicina. Rev Assoc Med Bras. 2005;51(1):23-8.

7. Avancini MT, Jorge M. Medos, atitudes e convicções de estudantes de medicina perante as doenças. 2001. Psiquiatria na prática médica UNIFESP. [acesso em Acesso em: 10 fev. 2010]. Disponível em: <http://www.unifesp.br/dpsiq/ polbr/ppm/original01.htm.>.

8. Araújo D, Peixinho, A. L. Qualitative evaluation in medicine: an experiment in medical propaedeutics at UFBA, 2003. Rev Bras Educ Med. 2006;30(2):20-30.

9. Crandall SJS, Volk RJ, Loemker V. Attitudes of medical students toward providing care for the underserved. J Am Med Assoc. 1993;269(9):2519-23.

10. Shorr F, Hayes RP, Finnerty JF. The effect of a class in medical ethics on fist year medical students. Acad Med. 1994;69(12):998-1000.

11. Hojat M, Vergare, M, Maxwell K, Brainard G, Herrine S, Isemberg $\mathrm{G} A$ et al. The devil is in the third year: a longitudinal study of erosion of emphaty in medical school. Acad Med. 2009;84(9):1182-91.

12. Mendonça AC, Villar HC, Tsuji SR. O conhecimento dos estudantes da faculdade de medicina de Marília (Famema) sobre responsabilidade profissional e segredo médico. Rev Bras Educ Med. 2009; 33(2):221-9. 
13. Quintana AM, Arpini DM. A atitude diante da morte e seu efeito no profissional de saúde: Uma lacuna da formação? Rev Psicologia Argumento. 2002;19(30):45-50.

14. Lind G. Moral regression in medical students and their learning environment. Rev Bras Educ Med. 2000;24(3):24-33.

15. Ribeiro MMF, Amaral CFS. Medicina centrada no paciente e ensino médico: a importância do cuidado com a pessoa e o poder médico. Rev Bras Educ Med. 2008; 32(1):90-7.

16. Ribeiro MMF. Avaliação da atitude do estudante de medicina da Universidade Federal de Minas Gerais a respeito da relação médico-paciente no decorrer do curso médico. Belo Horizonte; 2006. Doutorado [Tese] — Universidade Federal de Minas Gerais.

17. Silva RMFL, Rezende NA. O ensino de semiologia médica sob a visão dos alunos: implicações para a reforma curricular. Rev Bras Educ Med. 2008;32(1):32-9.

18. Lifshitz A, Gonzalez NJ. El médico ante los avances de la comunicación. Rev Med IMSS. 2000; 38(1):3-10.

19. Sousa ACJ, Tajra CRM, Coelho RS, Gomes CM, Teixeira RA. Ensino médico em um hospital privado: perspectiva dos pacientes e acompanhantes. São Paulo M J. 2009;127(2):101-4.

20. Brandon B, Spevak TA, Kassapedis E. Impact of Bedside Teaching Rounds on Patient Perception of Care. New York Medical Journal. 2008;1(2):45-51.
21. Lehman S, Brancati FL, Chen MC, Roter D, Dobs AS. The effect of bedside case presentations on patients' perceptions of their medical care. N Eng J Med.1997;336(16):1150-5.

\section{CONTRIBUIÇÃO DOS AUTORES}

Rilva Lopes de Souza-Muñoz, Isabel Barroso Augusto Silva e José Luis Simões Maroja contribuíram na concepção e desenho do estudo, aquisição, análise e interpretação dos dados elaboração da versão inicial do artigo, revisão crítica do conteúdo intelectual e aprovação da versão final.

\section{CONFLITO DE INTERESSES}

Declarou não haver.

\section{ENDEREÇO PARA CORRESPONDÊNCIA}

Rilva Lopes de Sousa-Muñoz

Departamento de Medicina Interna / Hospital Universitário Lauro Wanderley — UFPB

Campus I, s/n

Cidade Universitária UFPB — João Pessoa

CEP. 58050-000 PB

E-mail: dmi@ccm.ufpb.br 